\title{
Finite element analysis of the stress distribution in a representative elementary volume of mineral casting
}

\author{
Mihai Ciupan* \\ Department of Manufacturing Engineering, Technical University of Cluj-Napoca, Bld. Muncii no. \\ 103-105, 400641, Cluj-Napoca, Romania
}

\begin{abstract}
Mineral casting is a type of polymer concrete that has started to be used in commercial machine-tools in the last few years because of its low cost of manufacturing and vibration damping capabilities. The paper presents the modelling and finite element analysis of a representative elementary volume (REV) made of mineral casting subjected to tensile loading. The aim of the paper is to assess the stress distribution that develops inside the specimen in order to understand and improve the material.
\end{abstract}

\section{Introduction}

Some of the most modern machine-tools use structural elements such as beds, columns and tables made of mineral casting which is a type of polymer concrete [1]. An important first step in designing such elements is determining one or more compositions of mineral casting which optimize a number of given properties [2]. Typical properties which are of interest in machine-tool building are stiffness, vibration damping, and strength and not least, cost [3].

The simplest method to find a material that offers good results for all the specified properties is to experiment with different compositions. This involves the following steps:

- mixing different types and percentages of aggregates with thermosetting resins;

- $\quad$ casting samples and compacting them by vibrations;

- letting the samples cure, usually for a few days, in order to attain their final properties;

- $\quad$ testing their mechanical properties;

- plotting the results obtained for all the materials and interpolating them to obtain an optimum combination of properties for a given application.

As it can easily be seen, this is a very expensive and time-consuming method of determining which compositions have the required properties. It involves procuring different types of aggregates and resins, separating the aggregates based on size and having

${ }^{*}$ Corresponding author: mihai.ciupan@gmail.com 
specialized laboratory equipment such as a vibrating table and a bending or tensile testing machine.

A different method of finding an optimum composition involves building 3D representations of the material, analyzing them with the use of finite element methods and plotting and interpolating the results. While this method might lack some of the accuracy of the experiment, it provides good results at minimal costs, is time efficient and offers in depth information about the stresses and deformations that arise throughout the material.

The paper's objective is to develop and test a process through which a $3 \mathrm{D}$ representation of mineral casting, called a representative elementary volume (REV), can be built and its mechanical properties analyzed.

\section{Methods of representing the mineral casting structure}

The structure of the mineral casting can be modeled by representing aggregates as spheres with the rest of the space taken by the resin. The spheres approximate the irregular shapes of the minerals and have diameters which correspond to the sieve sizes. It is also important to maintain the same percentage of spheres of a certain size as in the composition that is to be analyzed.

In order to obtain accurate results, the REV should not have the spheres contact one another, but rather they should be separated by a small layer of resin. If the spheres touch one another, meshing will create a continuous body of mineral, instead of separate particles, and will result in a much higher tensile strength of the sample.

The REV can be constructed either by fitting different sized spheres in a given volume or by utilizing rigid body dynamics to simulate the action of mixing the aggregate and resin together [4]. The sphere fitting can be done manually or can be automated using different algorithms.

The advantage of using sphere fitting methods is shorter run times and simplicity. However, rigid body dynamics offer results that are closer to reality, with the smaller spheres clustering together just as in actual mineral casting [5].

The paper utilizes a method of manual sphere fitting due to its simplicity.

\section{Actual modelling of the REV}

The REV was modeled using the CAD software SolidWorks as an assembly consisting of aggregates which were represented as spheres placed inside a rectangular volume of resin. In order to simplify the 3D model and keep the number of parts to an acceptable limit, only the two largest particle sizes were used: $4 \mathrm{~mm}$ and $2 \mathrm{~mm}$ in diameter.

A small number of spheres were arranged so as to resemble a crystalline structure and a small gap was left between them so as to represent the resin matrix. This structure is shown in figure 1 with the gray sphere being $4 \mathrm{~mm}$ in diameter and the orange ones at $2 \mathrm{~mm}$.

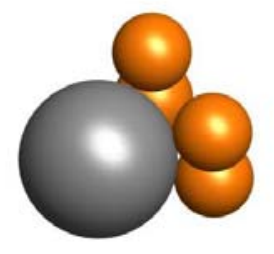

Fig. 1. Basic structure of the mineral particles. 
This structure was then patterned using predefined directions (Figure 2). The spheres which intersected the volume boundaries were discarded because SolidWorks was unable to generate a compatible mesh for these, meaning a mesh that has a node-to-node correspondence between the different entities. An incompatible mesh usually leads to higher than normal stresses and thus to simulation errors. The remaining spheres and the resin body can be seen in figure 3 .

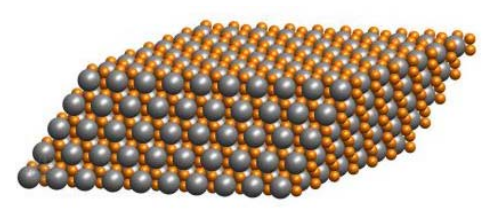

Fig. 2. Pattern made using the basic structure.

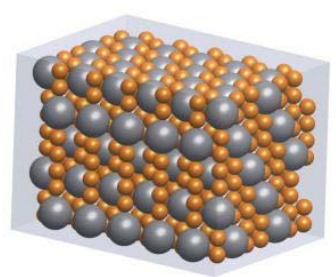

Fig. 3. REV composed of the mineral particles and a rectangular body of resin.

After arranging the spheres inside the volume, the resin matrix was constructed by subtracting the sphere bodies from the rectangular volume body. This was done by saving the assembly as a part and using the combine feature. Figure 4 shows a schematic of the subtraction operation. The next step implied editing the assembly again, removing the initial volume body and replacing it with the resin matrix part, which was previously built. The assembly modified as so had no intersecting parts, all the spheres were whole and the REV was obtained. The masses and mass percentages of the mineral casting constituents are given in Table 1.

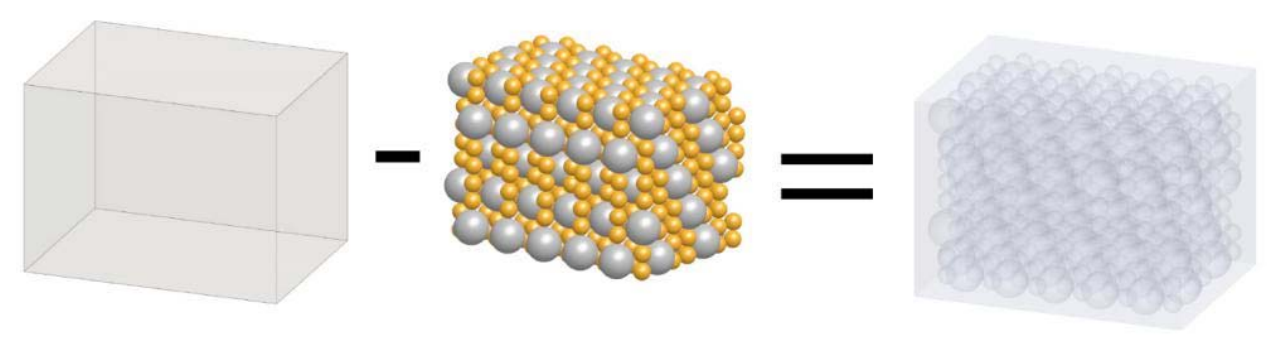

Fig. 4. The resin matrix.

Table 1. Mass properties of the REV constituents.

\begin{tabular}{|l|l|l|}
\hline & Mass [g] & Mass percentage [\%] \\
\hline Epoxy resin & 11.000 & 53.3 \\
\hline 4 mm spheres & 6.402 & 31.0 \\
\hline $2 \mathrm{~mm}$ spheres & 3.218 & 15.7 \\
\hline REV & 20.620 & 100.0 \\
\hline
\end{tabular}




\section{Finite element analysis of the REV}

The finite element analysis of the REV was conducted using a SolidWorks add-in: SolidWorks Simulation. Even though a nonlinear analysis would have been more appropriate, a linear one was selected instead due to the inconsistency of the material data found in the literature. Each of the bodies was assigned its specific material properties. Granite was chosen for the spheres representing the aggregates and epoxy resin for the matrix. Their respective properties can be found in Table 2 .

Table 2. Material properties.

\begin{tabular}{|l|l|l|}
\hline & Granite & Epoxy resin \\
\hline Elastic Modulus [GPa] & 70 & 2.415 \\
\hline Poisson's Ratio [] & 0.25 & 0.35 \\
\hline Mass Density [kg/m $\left.{ }^{3}\right]$ & 2900 & 1100 \\
\hline Tensile Strength [MPa] & 39 & 28 \\
\hline Compressive Strength [MPa] & 2200 & 104 \\
\hline
\end{tabular}

The contact between the components was set to bonded, meaning that there could be no relative movement between the body boundaries. The REV boundary to the left was fixed and a tensile force was applied to the one on the right (Figure 5).

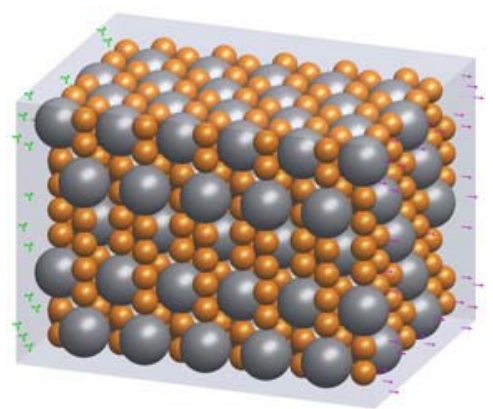

Fig. 5. The REV fixed on the left side and with a tensile force acting on the right side.

The REV was meshed using an element size of $1 \mathrm{~mm}$ and a tolerance of $0.05 \mathrm{~mm}$. This, coupled with the option of compatible meshing, resulted in a high quality mesh which was able to provide accurate analysis results and show the stress distribution even around small features.

The force pulling on the right side was incrementally increased until one of the bodies that make up the REV exhibited a stress approximately equal to its tensile strength (Figure 6). A force of $4500 \mathrm{~N}$ was obtained and it was divided to the section surface resulting in a tensile strength of $10 \mathrm{MPa}$ for the mineral casting. Figure 7 and 8 show points of high stress which develop in the resin between the mineral particles.

Young's modulus was also calculated based on the displacement plot section (Figure 9) at a value of $4.225 \mathrm{GPa}$, close to that of the epoxy resin. 


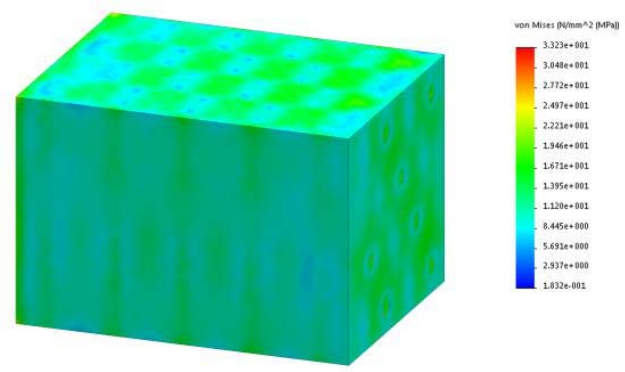

Fig. 6. Stress results for a tensile force of $4500 \mathrm{~N}$.

Figures 7 and 8 show that the highest stresses are encountered where the small particles are bound to the resin matrix. These seem to be the places where cracks initiate and ultimately lead to material failure.
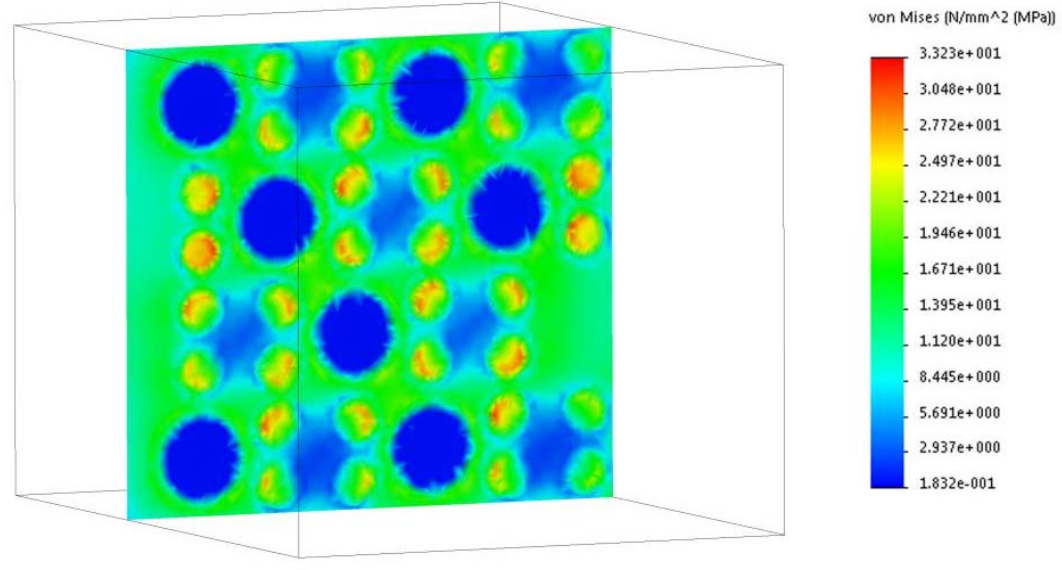

Fig. 7. Section through REV showing points of high stress.

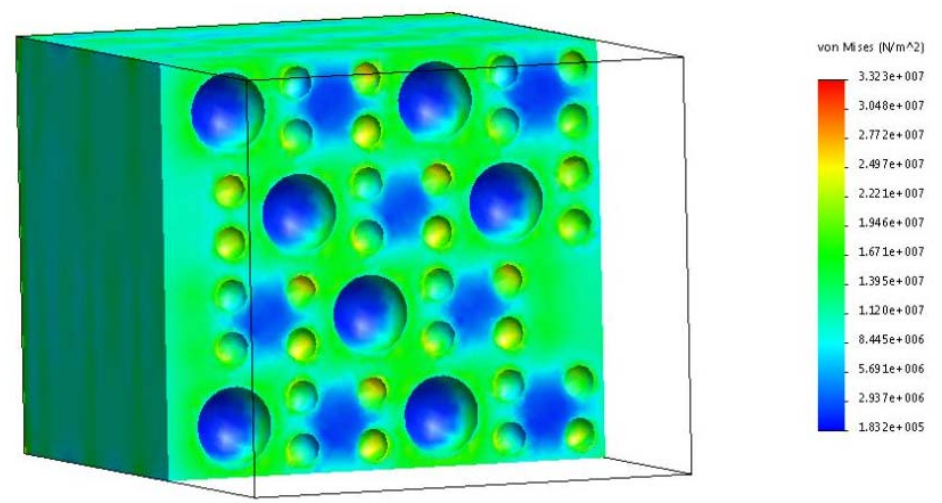

Fig. 8. Section through the stress plot of the resin matrix. 

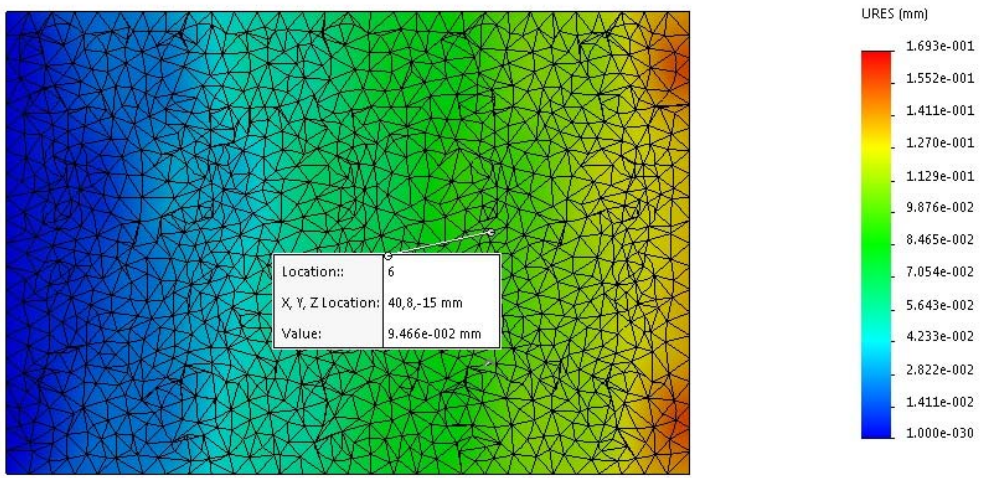

Fig. 9. Section through displacement plot with value listed at $40 \mathrm{~mm}$ from the fixed side on the left.

According to paper [6], the average values obtained for their composition of mineral casting were $18.42 \mathrm{MPa}$ for tensile strength and $29.738 \mathrm{GPa}$ for Young's modulus. The values predicted by the finite element analysis come close to the experimental values, but smaller. This may be due to a number of factors: the very large percentage of resin used in the 3D model, the fact that only large mineral particles were used and also the fact that the shape of the real mineral particles is different from spherical and in total have much more surface area for bonding with the epoxy.

\section{Conclusions}

This approach represents a method of finding the mechanical properties of a specific composition of mineral casting using numerical simulations. The method has some disadvantages. It requires manually redoing the aggregate pattern when different percentages of granular fractions are investigated. It also yields slightly different mechanical properties than in reality because of certain simplifications used.

However this method provides an easy, cost-effective and fast way of checking the mechanical properties of different mineral casting compositions. Additionally more information can be gained by seeing how the stresses develop and concentrate in different areas of the material.

Modelling and simulating a REV can be used over and over again for different materials or percentages of granular fractions in order to determine the optimum material for a given application, whether this means a higher Young's modulus, a higher tensile strength or better vibration damping.

\section{Future directions}

Future directions of research include using rigid body dynamics and random particle generation in order to automatically model the REV. This would lead to the automatic creation of REVs, thus speeding the process, and would enable testing a wide range of samples quickly in order to find the optimum for a specific application.

Simulation of the mixing of aggregates and resin using rigid body dynamics would enable obtaining 3D models which mimic real samples better and could offer information on how much mixing is required to obtain a somewhat uniform sample. 


\section{References}

1. N. Kepczak, W. Pawlowski. Application of Mineral Casting for Machine Tools Beds. Mechanics and Mechanical Engineering, 17(4), 285-289. (2013)

2. M. Ciupan. The design of composite structural components for a CNC machining center. Technical University of Cluj-Napoca (Master's dissertation). (2016)

3. M. Ciupan. 1st report for PhD program. Technical University of Cluj-Napoca. (2017)

4. B. Banerjee. Effective elastic moduli of polymer bonded explosives from finite element simulations. Cornell University Library. (2008)

5. P Stroeven, P. Stroeven. Assessment of Particle Packing Characteristics at Interfaces by Space System. Image Anal. Stereol, 19, 85-90. (2000)

6. N. Kepczak, W. Pawlowski, W. Blazejewski. The Study of the Mechanical Properties of the Mineral Cast Material. Archives of Mechanical Technology and Automation, 34(2), 25-32. (2014) 